\title{
BMJ Open Optic nerve sheath diameter sonography for the diagnosis of increased intracranial pressure: a systematic review and meta-analysis protocol
}

\author{
Alex Koziarz, ${ }^{1}$ Niv Sne, ${ }^{2}$ Fraser Kegel, ${ }^{2}$ Waleed Alhazzani, ${ }^{3}$ Siddharth Nath, ${ }^{1}$ \\ Jetan H Badhiwala, ${ }^{4}$ Timothy Rice, ${ }^{5}$ Paul Engels, ${ }^{6}$ Faidi Samir, ${ }^{2}$ Andrew Healey, ${ }^{7}$ \\ Kamyar Kahnamoui, ${ }^{8}$ Laura Banfield, ${ }^{9}$ Sunjay Sharma, ${ }^{1}$ Kesava Reddy, ${ }^{1}$ \\ Gregory W J Hawryluk, ${ }^{10}$ Andrew W Kirkpatrick, ${ }^{11}$ Saleh A Almenawer ${ }^{1}$
}

To cite: Koziarz A, Sne N, Kegel F, et al. Optic nerve sheath diameter sonography for the diagnosis of increased intracranial pressure: a systematic review and metaanalysis protocol. BMJ Open 2017;7:e016194. doi:10.1136/ bmjopen-2017-016194

- Prepublication history for this paper is available online. To view these files please visit the journal online (http://dx.doi org/10.1136/bmjopen-2017016194).

Received 31 January 2017 Revised 2 May 2017 Accepted 10 May 2017

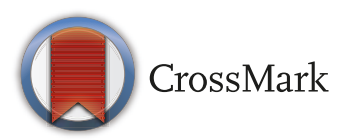

For numbered affiliations see end of article.

Correspondence to

Dr Saleh A Almenawer;

dr_menawer@hotmail.com

\section{ABSTRACT}

Introduction Increased intracranial pressure (ICP) is a significant neurological issue that may lead to permanent neurological sequelae. When evaluating patients with traumatic brain injury, it is crucial to identify those with high ICP in order to expedite ICP lowering measures and maintain adequate cerebral perfusion. Several measures are used to recognise patients with increased ICP including CT scan, MRI, ICP monitor, and lumbar puncture (LP). However, these tests can be invasive, associated with radiation exposure, contraindicated, or not readily available. Ultrasonography measurement of the optic nerve sheath diameter (ONSD) is proposed as a non-invasive and quick measure to identify high ICP. The aim of this systematic review and meta-analysis will be to examine the accuracy of ONSD sonography for increased ICP diagnosis.

Methods and analyses We will include published and unpublished randomised controlled trials, observational studies, and abstracts, with no publication type or language restrictions. Search strategies will be designed to peruse the MEDLINE, Embase, Web of Science, WHO Clinical Trials, ClinicalTrials.gov, CINAHL, and the Cochrane Library databases. We will also implement strategies to search grey literature. Two reviewers will independently complete data abstraction and conduct quality assessment. Included studies will be assessed using the Quality Assessment of Diagnostic Accuracy Studies-2 (QUADAS-2) tool. We will construct the hierarchical summary receiver operating characteristic curve for included studies and pool sensitivity and specificity using the bivariate model. We also plan to conduct prespecified subgroup analyses to explore heterogeneity. The overall quality of evidence will be rated using Grading of Recommendations, Assessment, Development and Evaluations (GRADE).

Ethics and dissemination Research ethics board approval is not required for this study as it draws from published data and raises no concerns related to patient privacy. This review will provide a comprehensive assessment of the evidence on ONSD sonography diagnostic accuracy and is directed to a wide audience. Results from the review will be disseminated extensively

\section{Strengths and limitations of this study}

- This study will investigate the diagnostic accuracy of optic nerve sheath diameter (ONSD) sonography for increased ICP across all available patient populations, reference standards, and covariates without publication type restriction.

- A comprehensive literature search, developed in consultation with a librarian with experience in systematic review search strategies, will be performed to include studies from multiple databases without language type restriction.

- Heterogeneity may exist due to potential multiple covariates; however, we plan to explore this using prespecified subgroup analyses.

- The current study protocol does not plan to evaluate cost-effectiveness and economic analysis when using ONSD sonography.

through conferences and submitted to a peer-reviewed journal for publication.

PROSPERO registration number CRD42017055485. Clinical trial number Trial registration number is NCT00783809.

\section{INTRODUCTION}

Increased intracranial pressure (ICP) is a significant neurological issue that may lead to permanent neurological sequelae. ${ }^{1}$ It can arise from head injury, intracranial mass lesion, disturbance of cerebrospinal fluid (CSF) circulation, obstruction to major venous sinuses, or occasionally be idiopathic. ${ }^{2}$ When evaluating patients with traumatic brain injury, it is crucial to identify those with high ICP in order to expedite ICP lowering measures and maintain adequate cerebral perfusion. ${ }^{3}$ Several measures are used to recognise patients with increased ICP including CT scan, MRI, ICP monitor, and 
lumbar puncture (LP). However, these tests can be invasive, associated with radiation exposure, contraindicated, or not readily available. ${ }^{4}$ Ultrasonography measurement of the optic nerve sheath diameter (ONSD) is proposed as a non-invasive and quick measure to identify high ICP. The optic nerve is wrapped by a sheath derived from the meninges and extends towards the orbit. ${ }^{5}$ This communication allows CSF to transfer and therefore have similar pressure changes between the intracranial and intraorbital subarachnoid spaces. This method has been proposed in the setting of trauma, neurosurgery, and emergency medicine. ${ }^{67}$

The aim of this systematic review and meta-analysis protocol will be to examine the accuracy of ONSD sonography for the diagnosis of increased ICP. This systematic review and meta-analysis protocol is designed in accordance to the Preferred Reporting Items for Systematic reviews and Meta-Analysis Protocols (PRISMA-P) ${ }^{8}$ and is registered with the International Prospective Register of Systematic Reviews (PROSPERO; CRD42017055485).

\section{METHODS}

We will conduct a systematic review and meta-analysis in accordance with the Preferred Reporting Items for Systematic Reviews and Meta-Analyses (PRISMA) ${ }^{89}$ and the Cochrane Handbook for Systematic Reviews of Diagnostic Test Accuracy. ${ }^{10}$ This protocol will be amended and updated in conjunction with the PRISMA-P guidelines. Updated versions will be made available on PROSPERO with record of version history.

\section{Literature search}

A detailed librarian-assisted search will be conducted of the MEDLINE, Embase, Web of Science, WHO Clinical Trials, ClinicalTrials.gov, and the Cochrane Library databases from inception to an update on June 5, 2017 to ensure all recent relevant studies are captured. We will include published and unpublished reports on the diagnostic accuracy of ONSD in detecting increased ICP without language or publication type restrictions. This search will be developed in collaboration with a librarian who has experience in conducting searches for systematic reviews and meta-analyses. ${ }^{11}$ Keywords and medical subject heading (MeSH) terms related to optic nerve, sonography, and ICP will be used. The search strategy utilised for MEDLINE is provided in table 1 . This search will be supplemented by manually reviewing the references of included articles. We will search for grey literature, which may include dissertations, reports, and conference abstracts. Experts will be contacted for potential eligible studies.

\section{Study selection}

Obtained studies from the literature search will be independently evaluated by two investigators (AK, FK) for eligibility. Disagreements between reviewers regarding whether to include or exclude a study will be resolved by
Table 1 Search strategy for the MEDLINE electronic database using the Ovid interface

\begin{tabular}{|c|c|}
\hline Database & Search Terms \\
\hline \multirow{30}{*}{$\begin{array}{l}\text { MEDLINE } 1946 \text { - } \\
\text { present }\end{array}$} & 1. optic nerve/ \\
\hline & 2. optic nerve*.ti,ab,kf. \\
\hline & 3. nervus opticus.ti,ab,kf. \\
\hline & 4. second cranial nerve*.ti,ab,kf. \\
\hline & 5. cranial nerve ii.ti,ab,kf. \\
\hline & 6. cranial nerve 2.ti,ab,kf. \\
\hline & 7. or/1-6 \\
\hline & 8. oligodendroglia/ or myelin sheath/ \\
\hline & 9. sheath.ti,ab,kf. \\
\hline & 10. oligodendroglia.ti,ab,kf. \\
\hline & 11. oligodendrocyte*.ti,ab,kf. \\
\hline & 12. myelin.ti,ab,kf. \\
\hline & 13. or/8-12 \\
\hline & 14. diameter*.ti/ab/kf. \\
\hline & 15. diametre*.ti,ab,kf. \\
\hline & 16. dilat*.ti/ab/kf. \\
\hline & 17. thick*.ti,ab,kf. \\
\hline & 18. volume.ti,ab,kf. \\
\hline & 19. (swelling or swollen).ti,ab,kf. \\
\hline & 20. oedema.ti,ab,kf. \\
\hline & 21. or/14-20 \\
\hline & 22. intracranial pressure/ \\
\hline & $\begin{array}{l}\text { 23. ((intracranial or subarachnoid*) adj2 } \\
\text { pressure).ti,ab,kf. }\end{array}$ \\
\hline & 24. intracranial hypertension/ \\
\hline & 25. intracranial hypertension.ti,ab,kf. \\
\hline & 26. icp.ti,ab,kf. \\
\hline & 27. papilledema/ \\
\hline & 28. papilledema*.ti,ab,kf. \\
\hline & 29. or/22-28 \\
\hline & 30. (7 or 13) and 21 and 29 \\
\hline
\end{tabular}

consensus and a third reviewer (SAA) will be consulted, if necessary. Inclusion criteria for this study will include:

- Study design: Randomised controlled trials (RCTs; including pilot studies), controlled (non-randomised) clinical trials, prospective and retrospective cohort studies, and abstracts. There will be no restriction to publication type or language.

- Population: Patients of any age group and demographic with suspected increased ICP.

- Index test: ONSD sonography.

- Reference standard: CT scan, MRI, ICP monitor, or LP.

For studies that were published more than once, only the most recent and comprehensive report will be included. Studies that did not include a reference standard, studies without clinical outcomes including in vitro studies, review 


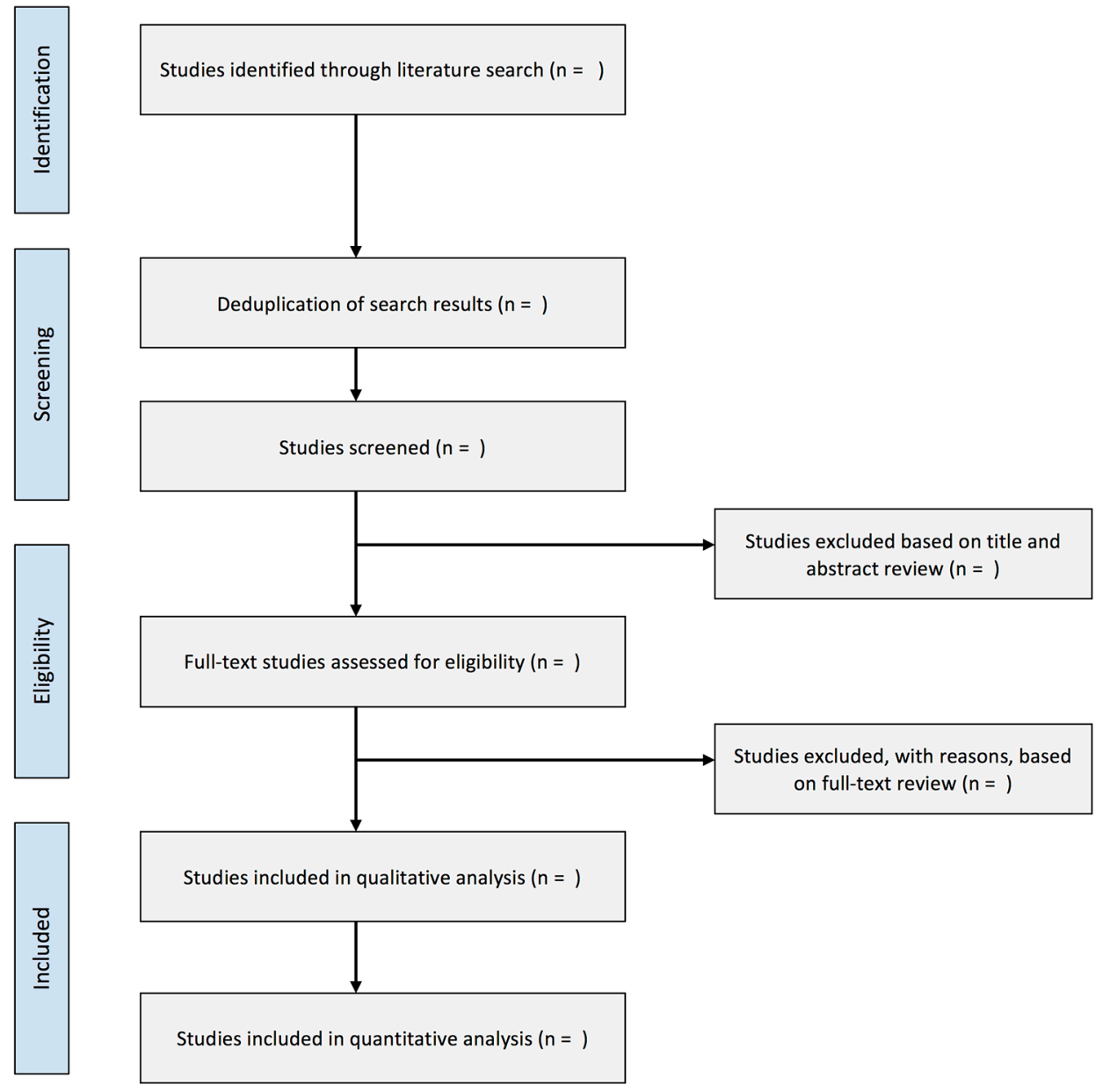

Figure 1 Flowchart of study selection.

articles, letters, and correspondences or comments will be excluded (figure 1).

\section{Monitoring of ocular nerve sheath in traumatic increased ICP (MOONSTRIP) study (NCT00783809)}

In addition, we will present and then include the MOONSTRIP unpublished prospective blinded study conducted at a level one tertiary care trauma centre. Investigators of this study were given visual materials and readings prior to attending didactic and hands-on supervised ultrasound training sessions. During these sessions, quality assurance data were collected to ensure all investigators met acceptable standards.

In this study, all patients referred to the trauma centre will undergo assessment in accordance with advanced trauma life support protocol. If assessment warrants investigation for increased ICP, a CT scan will be performed. An investigator blinded to the CT scan will assess the patient based on the following inclusion criteria: age $\geq 16$ years, following trauma, and ONSD sonography required to be performed within 1 hour of the CT scan. All patients will be consented prior to enrolment. Exclusion criteria includes: penetrating trauma to the head or significant ocular trauma; patient not expected to survive transfer out of emergency room department; patient too unstable to undergo CT of head or ONSD sonography.

ONSD sonography of the eye will be conducted by an investigator blinded to CT scan findings. The investigator will use an ultrasound machine with a linear transducer. A layer of sterile coupling gel will be applied to the closed eyelid with the patient in supine position. ONSD sonography will be conducted $3 \mathrm{~mm}$ posterior to the globe for each eye. Transverse and sagittal ONSD measurements of each eye will be averaged and recorded for subsequent inter-observer variability analysis.

The reference test for this study is CT scan, which will be reviewed by a neuroradiologist blinded to the ONSD sonography findings. The presence of elevated ICP will be based on the following criteria: midline shift from mass effect of $3 \mathrm{~mm}$ or greater, collapse of third ventricle, hydrocephalus, effacement of sulci with evidence of significant oedema, collapse of mesencephalic cisterns, or evidence of herniation. ${ }^{12}$ The patient will be followed up by the research coordinator who is blinded to sonography and CT findings for a duration of 48 hours after ONSD sonography. During this period if the patient needs a repeat CT of the head or any intervention that would require 
the insertion of an ICP monitor, repeat measurements of the ONSD will be performed. Patients demographics will be evaluated including age, Glasgow Coma Scale (GCS), mechanism of injury, Injury Severity Score (ISS), whether patient is intubated, time from presentation to CT scan, CT findings, haemodynamic parameters, monocular and binocular ONSD. This patient information will be stored in a confidential encrypted database. The inter-observer variability will be calculated using the kappa statistic for the left eye, right eye, and binocular average of ONSD measurements. In addition, the ONSD sonography accuracy will be calculated using sensitivity, specificity, positive and negative predictive value, and positive and negative likelihood ratio.

\section{Data management and collection}

Literature search results will be downloaded from databases as. ris files or. ciw files which will contain the complete reference and loaded into EndNote X7 for reference management. Reviewers will develop and pilot screening questions and forms based on the eligibility criteria. Full text articles will be retrieved before data extraction. For studies not published in English, the full text will be translated and a medical expert fluent in the original language of the article will be contacted to assist with data abstraction. We will retrieve the full text of each article that meets eligibility criteria or that contains ambiguity. If necessary, the authors of potentially relevant studies will be contacted for missing data or to resolve questions about eligibility. Discrepancies will be resolved by consensus. A third author will be consulted in the event of a disagreement. Reasons for article exclusion will be recorded.

Two reviewers will independently extract data from eligible articles, which will be verified by a third reviewer. Data collection forms will contain fields for variables, such as: study design; study first author; year of publication; journal of publication; language; countries and years of patient recruitment; sample size; number of males and females; sample mean age; sample age cut-off for inclusion; patient population with high ICP (eg, trauma or brain pathology) and units of measurement for high ICP ( $\mathrm{mm} \mathrm{Hg}$ or $\mathrm{cm} \mathrm{H}_{2} \mathrm{O}$ ); patient diagnosis; index and reference test specifications; number of trained sonographers in a study; medical specialty of sonographer; GCS; number of ONSD measurements; monocular versus binocular ONSD measurement; timing of ONSD measurement; reference test findings; ONSD cut-off for high ICP and whether this cut-off was determined a priori or calculated as post hoc; time interval between conducting ONSD sonography and reference standard; number of true and false positives, true and false negatives; sensitivity and specificity; positive and negative predictive value; positive and negative likelihood ratio; area under receiver operating characteristic (ROC) curve.

\section{Risk of bias in individual studies}

Two reviewers will independently perform quality assessment. Risk of bias will be assessed using the Quality
Assessment of Diagnostic Accuracy Studies-2 (QUADAS-2) tool $^{13}$ to evaluate the risk of bias and applicability of studies across four domains: patient selection, index test, reference standard, and flow and timing. Each domain is evaluated for risk of bias with the first three domains evaluated based on applicability. In the instance of unresolved disagreement, a third reviewer will be consulted.

\section{Index test}

The optic nerve sheath is contiguous with the dura mater, and the CSF contained within it is contiguous with the subarachnoid space surrounding the brain and spinal cord. As such, raised ICP can be visualised sonographically by increased ONSD. ${ }^{14}$ Ultrasound examinations are typically conducted with a linear array ultrasound transducer with a layer of sterile coupling gel applied to the closed eyelid while the patient is positioned supine. The ONSD is measured $3 \mathrm{~mm}$ posterior to the globe for each eye. ${ }^{14-16}$ The cut-off of ONSD measurement to diagnose high ICP used in individual studies is either determined a priori or post-hoc analysis after calculating the area under the ROC curve.

\section{Reference standard}

Several reference tests are used for the diagnosis of increased ICP which may include CT scan, MRI, invasive ICP monitoring, or LP. CT scan can diagnose increased ICP and determine its cause by identifying mass lesion, cerebral oedema, midline structural shift, ventricular collapse or enlargement, and cistern compression. ${ }^{12}$ Due to cost concerns and relatively minimal added value of MRI in detecting high ICP beyond CT scan findings, ${ }^{17}$ MRI is not used as common as CT to diagnose patients with high ICP. Invasive ICP monitoring may include intraventricular or intraparenchymal catheters, and subdural or subarachnoid bolts. A raised ICP is generally defined as pressure greater than $22 \mathrm{~mm} \mathrm{Hg}$ that warrants treatment. ${ }^{18}$ These devices involve creating a burr-hole and insertion of the ICP monitor either in the ventricular system, brain parenchyma, subdural, or subarachnoid space. LP can also be used for the diagnosis of increased ICP by measuring the opening pressure.

\section{Statistical analysis and data synthesis}

Sensitivity and specificity estimates will be used to produce a forest plot with corresponding $95 \%$ confidence intervals (CI) and a summary ROC (SROC) curve to explore between-study variation for the accuracy of ONSD sonography.

The sensitivity, specificity, positive and negative predictive value, and positive and negative likelihood ratios will be extracted from eligible studies with associated $95 \%$ CIs. If required, these measures will be calculated using $2 \times 2$ contingency table consisting of true and false positives and true and false negatives that will be reconstructed for each study. We will pool the summary sensitivity and specificity stratified according to reference standard using the bivariate mode ${ }^{19}$ with random-effects weighting. ${ }^{10}$ This model for diagnostic 
meta-analysis accounts for between-study variability when pooling sensitivity and specificity.

We will also perform meta-analysis using the hierarchical summary receiver operating characteristic (HSROC) model ${ }^{20}$ with random-effects weighting to obtain the SROC curve and determine if the summary point is an accurate predictor of raised ICP. ${ }^{10}$ The HSROC model includes two parameters that account for between-study variation regarding accuracy and threshold level, and a shape parameter that allows for non-symmetrical underlying summary ROC curve. For this model, we will combine all eligible studies regardless of thresholds and reference standards used. We shall use this model to explore heterogeneity by examining how the curve's position and shape may alter with study level covariates. ${ }^{10}{ }^{21}$ The magnitude of heterogeneity will be depicted by approximating the degree of proximity of observed study results to the SROC curve. We will also indicate how much greater the $95 \%$ prediction regions of the SROC curve are compared with the $95 \%$ confidence regions. ${ }^{22}$ High heterogeneity will be noted when the $95 \%$ prediction regions are much larger graphically relative to the $95 \%$ confidence regions. ${ }^{22}$ Meta-analyses will be performed using the NLMIXED procedure in the Statistical Analysis System (SAS) software package (version 9.3; SAS Institute Inc., Cary, North Carolina, USA).

Methods for evaluating publication bias in diagnostic accuracy studies are limited, and hence, results will be interpreted with caution. Potential publication bias will be investigated by funnel plot asymmetry inspection which will be constructed using Deeks model. ${ }^{23}$ Diagnostic odds ratio (DOR) will be calculated to evaluate potential publication bias using the following equation ${ }^{10}$ :

$$
D O R=\frac{T P / F P}{F N / T N}
$$

We will use likelihood ratios to determine post-test probabilities, which will be computed using these pre-test probabilities and the summary positive and negative likelihood ratios.

Covariates that may affect study heterogeneity and contribute to variance in index test diagnostic accuracy parameters will be explored. We plan to conduct prespecified subgroup analyses stratified by: quality of included study; patient population; reference standard; trained versus untrained sonographer; whether the sonographer was blinded or not from reference standard results; timing of ONSD sonography; whether this cut-off was determined a priori or calculated as post hoc. In addition, we will conduct sensitivity analysis by performing a meta-analysis including and excluding the MOONSTRIP study. Quality of evidence from the meta-analysis will be rated using the Grading of Recommendations Assessment Development and Evaluation (GRADE) methodology. ${ }^{24}$

\section{ETHICS AND DISSEMINATION}

Approval from the research ethics board is not required for this study as it examines data from conducted studies.
Therefore, there are no concerns regarding patient privacy or participant ethics. Results from this study are expected to significantly change clinical practice. The examination of the literature investigating the diagnostic accuracy of ONSD sonography for increased ICP is expected to inform clinicians in different fields of medicine, such as trauma, neurosurgery, and emergency medicine. The results from this review will be submitted to a peer-reviewed journal for publication and will be widely presented at conferences and seminars.

\section{Author affiliations}

${ }^{1}$ Division of Neurosurgery, McMaster University, Hamilton, Ontario, Canada ${ }^{2}$ Division of General Surgery and Trauma, McMaster University, Hamilton, Ontario, Canada

${ }^{3}$ Department of Medicine, McMaster University, Hamilton, Ontario, Canada ${ }^{4}$ Division of Neurosurgery, University of Toronto, Toronto, Ontario, Canada ${ }^{5}$ Division of General Surgery, McMaster University, Hamilton, Ontario, Canada ${ }^{6}$ Division of General Surgery, Trauma, and Critical Care, McMaster University, Hamilton, Ontario, Canada

${ }^{7}$ Division of Emergency Medicine, McMaster University, Hamilton, Ontario, Canada ${ }^{8}$ Division of General Surgery and Critical Care, McMaster University, Hamilton, Ontario, Canada

${ }^{9}$ Health Sciences Library, McMaster University, Hamilton, Ontario, Canada

${ }^{10}$ Department of Neurosurgery and Neurology, University of Utah, Salt Lake City, Utah, USA

${ }^{11}$ Department of Surgery and Critical Care, University of Calgary, Calgary, Canada

Contributors AK, NS, and SAA conceived and designed the protocol. AK, LB, and SAA designed the search strategy and piloted it across all relevant databases. AK, NS, FK, and SAA developed the review protocol, selection criteria, and risk of bias assessment. AK and SAA designed the data management and synthesis methodology. AK, NS, FK, WA, SN, JHB, TR, PE, FS, AH, KK, LB, SS, KR, GWJH, AWK, and SAA critically reviewed and collaborated in the discussion of the intellectual content of the manuscript.

Funding This research received no specific grant from any funding agency in the public, commercial, or not+for+profit sectors.

Competing interests None declared.

Provenance and peer review Not commissioned; externally peer reviewed.

Open Access This is an Open Access article distributed in accordance with the Creative Commons Attribution Non Commercial (CC BY-NC 4.0) license, which permits others to distribute, remix, adapt, build upon this work non-commercially, and license their derivative works on different terms, provided the original work is properly cited and the use is non-commercial. See: http://creativecommons.org/ licenses/by-nc/4.0/

(c) Article author(s) (or their employer(s) unless otherwise stated in the text of the article) 2017. All rights reserved. No commercial use is permitted unless otherwise expressly granted.

\section{REFERENCES}

1. Zanier ER, Ortolano F, Ghisoni L, et al. Intracranial pressure monitoring in intensive care: clinical advantages of a computerized system over manual recording. Crit Care 2007;11:R7.

2. Dunn LT. Raised intracranial pressure. Journal of Neurology, Neurosurgery \& Psychiatry 2002;73(suppl 1):i23-i27.

3. Joseph B, Haider AA, Pandit V, et al. Changing paradigms in the management of 2184 patients with traumatic brain injury. Ann Surg 2015;262:440-8.

4. Mayhall CG, Archer NH, Lamb VA, et al. Ventriculostomy-Related infections. N Engl J Med 1984;310:553-9.

5. Wang L, Feng L, Yao Y, et al. Optimal optic nerve sheath diameter threshold for the identification of elevated opening pressure on lumbar puncture in a Chinese population. PLoS One 2015;10:e0117939.

6. Amin D, McCormick T, Mailhot T. Elevated intracranial pressure diagnosis with Emergency Department Bedside ocular ultrasound Case Rep Emerg Med 2015;2015:1-3. 
7. Tayal VS, Neulander M, Norton HJ, et al. Emergency department sonographic measurement of optic nerve sheath diameter to detect findings of increased intracranial pressure in adult head injury patients. Ann Emerg Med 2007;49:508-14.

8. Liberati A, Altman DG, Tetzlaff J, et al. The PRISMA statement for reporting systematic reviews and meta-analyses of studies that evaluate healthcare interventions: explanation and elaboration. BMJ 2009;339:b2700.

9. Moher D, Liberati A, Tetzlaff $\mathrm{J}$, et al. Preferred reporting items for systematic reviews and meta-analyses: the PRISMA statement. BMJ 2009;339:b2535.

10. Macaskill P, Gatsonis C, Deeks JJ, et al. Chapter 10: Analysing and Presenting Results. In: Deeks JJ, Bossuyt PM, Gatsonis C, eds. Cochrane Handbook for Systematic Reviews of Diagnostic Test Accuracy Version 1.0. The Cochrane Collaboration, 2010. http:// srdta.cochrane.org/.

11. Sollenberger JF, Holloway RG. The evolving role and value of libraries and librarians in health care. JAMA 2013;310:1231-2.

12. Golshani K, Ebrahim Zadeh M, Farajzadegan Z, et al. Diagnostic accuracy of Optic Nerve Ultrasonography and Ophthalmoscopy in prediction of elevated intracranial pressure. Emerg 2015;3:54-8.

13. Whiting PF, Rutjes AW, Westwood ME, et al. QUADAS-2: a revised tool for the quality assessment of diagnostic accuracy studies. Ann Intern Med 2011;155:529-36.

14. Hansen HC, Helmke K. The subarachnoid space surrounding the optic nerves. an ultrasound study of the optic nerve sheath. Surg Radiol Anat 1996;18:323-8.

15. Hansen HC, Helmke K, Kunze K. Optic Nerve Sheath Enlargement in acute intracranial hypertension. Neuro-ophthalmology 1994;14:345-54.

16. Helmke K, Hansen HC. Fundamentals of transorbital sonographic evaluation of optic nerve sheath expansion under intracranial hypertension. I: experimental study. Pediatr Radiol 1996;26:701-5.

17. Said RR, Rosman NP. A negative cranial computed tomographic scan is not adequate to support a diagnosis of pseudotumor cerebri. J Child Neurol 2004;19:609-13.

18. Carney N, Totten AM, O'Reilly C, et al. Guidelines for the management of severe traumatic brain injury, Fourth Edition. Neurosurgery 2016:1 (Epub ahead of print).

19. Reitsma JB, Glas AS, Rutjes AW, et al. Bivariate analysis of sensitivity and specificity produces informative summary measures in diagnostic reviews. J Clin Epidemiol 2005;58:982-90.

20. Rutter CM, Gatsonis CA. A hierarchical regression approach to meta-analysis of diagnostic test accuracy evaluations. Stat Med 2001;20:2865-84.

21. Lee J, Kim KW, Choi SH, et al. Systematic review and Meta-Analysis of studies evaluating diagnostic test Accuracy: a Practical Review for clinical Researchers-Part II. statistical methods of Meta-Analysis. Korean J Radiol 2015;16:1188-96.

22. Bossuyt P B, Davenport C, Deeks J, et al. Chapter 11: Interpreting results and drawing conclusions. In: Deeks JJ, Bossuyt PM, Gatsonis C, eds. Cochrane Handbook for Systematic Reviews of Diagnostic Test Accuracy Version 0.9. The Cochrane Collaboration, 2013. http:// srdta.cochrane.org/.

23. Deeks JJ, Macaskill P, Irwig L. The performance of tests of publication Bias and other sample size effects in systematic reviews of diagnostic test accuracy was assessed. J Clin Epidemiol 2005;58:882-93.

24. Schünemann $H J$, Schünemann $A H, O x m a n A D$, et al. Grading quality of evidence and strength of recommendations for diagnostic tests and strategies. BMJ 2008;336:1106-10. 